\title{
A novel cooperative spectrum signal detection algorithm for underwater communication system
}

\author{
Jiang Xiaolin ${ }^{1,2^{*}} \mathbb{D}$, Tang Zhengyu ${ }^{2}$ and Wang Ronghui ${ }^{3}$
}

\begin{abstract}
In order to further improve the spectrum resource detection probability and increase the spectrum utilization rate in underwater wireless communication systems, this paper designs a novel multi-layer cooperative spectrum sensing algorithm based on compressed sensing, which uses compressed sensing technology to estimate the spectrum to reduce the sampling rate and the overhead of sonar signals. This new algorithm seeks the optimal hyper-parameter through Bayesian model. The multi-layer Bayesian model is introduced into the Dirichlet process to realize the automatic grouping of compressed perceptual data with the information from the non-parametric grouping mechanism, and the optimal super-parameters are selected through the fusion center to determine the spectrum. Simulation results show that the proposed algorithm fully considers the temporal correlation of compressed perceptual data and effectively improves spectral sensing performance of underwater communication system.
\end{abstract}

Keywords: Signal detection algorithm, Compressed sensing, Underwater communication

\section{Introduction}

With the increase of people's demand for underwater communication, underwater wireless communication has been widely concerned by researchers. At present, underwater communication usually adopts sound wave as the carrier of signal transmission. Sound wave frequency is low, bandwidth is narrow, and underwater communication environment is very complex. The available communication spectrum of an underwater acoustic channel with serious attenuation of a wireless electromagnetic wave is quite limited. On the other hand, a large amount of communication data and high sampling rate aggravate the scarcity of spectrum resources in underwater acoustic communication. In addition, in underwater communication, more and more communication applications require that channels provide wider frequency bands. How to effectively realize fast perception of spectrum resources and realize broadband information transmission? Some researchers have proposed

\footnotetext{
* Correspondence: 57102526@qq.com

${ }^{1}$ Harbin Institute of Technology, Harbin 150000, Heilongjiang, China

${ }^{2}$ Heilongjiang University of Science and Technology, 150000, Harbin, China Full list of author information is available at the end of the article
}

to introduce compressed sensing technology into spectrum perception. Compressive sensing technology has a huge application prospect. In literature [1], Zhi et al. first proposed the application of compressive sensing theory to cognitive radio, using wavelet edge detection and base tracking algorithm for spectrum edge detection. Literature [2] makes full use of L1 norm to solve the problem and reconstructs the signal by minimizing the L1 norm problem. For the L1 norm problem, a simple matching pursuit (MP) algorithm was proposed in literature [3]. In literature [4], orthogonal matching pursuit (OMP) algorithm is proposed on the basis of MP. In literature [5], on the basis of OMP, a piecewise orthogonal matching pursuit (StOMP) algorithm is proposed, which compares selected atoms with a threshold, updates the residual values, and iterates through a loop to get the final solution. The compressed sensing process not only reduces the limitation of signal bandwidth, but also increases the computational complexity and spectrum sensing time of the system due to the complexity of signal reconstruction. The researchers are also working on different ways to reduce computational complexity and improve perceptual performance. In 
literature [6], sparse Bayesian learning is used to reconstruct the compressible image under the condition of noise measurement, and it is verified that this method can effectively reduce the time of spectrum perception. In reference [7], sparse Bayesian model and correlation vector machine learning were studied, and sparse Bayesian regression and classification model were established to reduce the complexity. In literature [8], CS is used for spectrum sensing, and CS data is studied to conduct joint data fusion of some users' compressed sensing data under the condition that CS data do not share the same sparse characteristics with super-parameters. Literature [9] proposes a Bayesian direct spectrum sensing algorithm, that is, Bayesian compressed sensing is used to detect the spectrum without reconstructing the signal, and the information of the main user is estimated directly from the over-parameter in the compressed measured value to complete the detection. This model can estimate not only the signal parameters, but also the error band of the signal.

Compressive sensing technology is a technique proposed for sparse signal sampling and compression at the same time. By sampling a very few characteristic observation values of analog signal, signal acquisition is completed through spatial transformation. The receiver completes the original signal reconstruction from the compressed data by solving an optimization problem. However, the signal reconstruction process in compressed sensing is relatively complex, which makes the computational complexity of the system large and the spectrum perception time long, and affects the overall performance of the system. In the process of spectrum signal detection, cognitive users only care about whether the spectrum is occupied or not, but do not care about which specific signal. In this paper, we use this feature to design a novel algorithm which is spectrum signal detection algorithm based on compressed sensing. In the underwater communication system, each SU is compressed and data acquisition, using Bayesian model to obtain shared super-parameters, fusion center through super-parameters to complete the decision of the spectrum occupancy information. Without signal reconstruction, a single SU completes spectrum signal detection and then popularized the algorithm to the whole underwater cognitive wireless communication system, making full use of the compressed sensing data collected by different cognitive users at different layer and fully considering the correlation of compressed sensing data to complete the signal detection process.

\section{System model and analysis}

\subsection{Methods}

If the sonar signal of the underwater communication system is a one-dimensional discrete-time signal with finite length and real value, it is expressed by $x$, which is a column vector of $N \times 1$ in $\mathrm{RN}$ space. If in $\mathrm{RN}$ there exists a base matrix of $N \times N$ which is composed of $N$ standard orthogonal bases $\psi=\left[\psi_{1}, \psi_{2}, \ldots, \psi_{N}\right]$, in which each column vector in the base matrix is $\psi_{i}\{i=1,2 \ldots$. $N\}$, a set of standard orthogonal bases for $N \times 1$, in which any discrete-time vector can be represented by this basis matrix, that is, the sonar signal $x$ can be expressed as the following:

$$
x=\psi s
$$

where $s$ is the weighted coefficient column vector of $N \times 1$. It can be seen from formula (1) that both $x$ and $s$ can indicate that the sonar signal to be compressed is in time domain, $x$ is in the time domain, frequency domain, or spatial domain, while $s$ is in the domain of $\psi$.

First, we define the sparsity of the sonar signal. If most of the observed coefficients of a signal are equal to zero or nearly zero, and only a few non-zero coefficients exist, then the sonar signal is sparse in this domain, which is called sparse domain. In the process of compression sensing, the most important condition is that the sonar signal is sparse in a certain domain, so how to select the sparse domain is the basis of realizing compression sensing. From the introduction, we can know that the number of authorized users is very small, and the spectrum utilization of some bands is very low. The signal of authorized users in cognitive radio network is sparse in the frequency domain, which satisfies the condition of compressed sensing application. Therefore, compressed sensing technology can be introduced to optimize the design. If there are only $K$ numbers belong to non-zero values in the column vector $s$, the other values are approximately equal to or equal to 0 , and satisfy the $K<<N$, at this point, the signal $x$ is sparse relative to the range $\psi$, satisfying the application condition of compression sensing. For $K$ sparse sonar signal $x$, the theory of compression sensing proves that $M$ sample values are selected in signal $x$ by measuring matrix, and the receiver can use $M$ sample values to realize signal reconstruction with high probability, in which $M$ is far less than $N$, so data compression can be realized. The acquisition of the $M$ sample values is represented by the following formula

$$
y=\Phi x=\Phi_{\Psi s}
$$

where $y$ is a vector of $M \times 1$, whose elements represent the size of the $M$ sample values extracted, and $\Phi$ is the measurement matrix of $M \times N . M / N$ is the compression ratio, which indicates the degree of compression of the data in the process of compression sensing. The sparse sonar signal information should not be lost during the compression of the measurement matrix, so the selection of the measurement matrix is strictly required, and 
scholars have made extensive research on the measurement matrix. The restricted isometry property (RIP) [10] of a matrix is a widely accepted constraint condition, that is for $K$ sparse sonar signals $x$. If there is a value $\delta_{k} \in$ $(0,1)$ such that:

$$
\left(1-\delta_{k}\right)\|x\|_{2}^{2} \leq\|\Phi x\|_{2}^{2} \leq\left(1+\delta_{k}\right)\|x\|_{2}^{2}
$$

It is called $\Phi$ satisfies the $K$ order RIP characteristic. The RIP parameter of order $K$ is $\delta_{k} \in(0,1)$. In practice, the $\delta_{k}$ smaller value means more stringent constraints and better reconfiguration performance [11].

\subsection{Collaborative awareness model for underwater communication system}

The underwater communication system structure is shown in Fig. 1.

In the underwater communication cognitive wireless network, a single-layered center is occupied by SU and PU. When signal detection is needed, the control center sends out an indication signal, and all members of the hierarchical center synchronously perform signal detection operations. Each SU collects compressed data, uses Bayesian model to obtain shared super-parameters, and transmits the results to the cognitive radio user base station to get the preliminary signal detection results. When the members of the hierarchical center collect the perceptual data, the distributed computation is carried out and the results are broadcast to the hierarchical control center [12, 13]. After several information exchanges, the proposed spectrum signal detection algorithm will approach a fixed value and obtain preliminary signal detection results. The next step is to fuse the data obtained from a single hierarchical center through the data fusion center to realize the joint signal detection of multiple hierarchical centers, because PU is also distributed in different locations in space and the transmission power is limited. The sparse state of the wireless spectrum may vary with the change of spatial position [14]. Therefore, the compressed perceptual data of different stratified centers may not be statistically relevant, and the super-parameters obtained may not be ideal. In this section, a hierarchical Bayesian model based on Dirichlet process (DP) is proposed to realize compressed sensing. The data are grouped and the shared hyper-parameters are mined in each group to achieve the optimal signal detection results.

\subsection{Bayesian analysis}

In the process of transmitting information underwater, there will inevitably be noise interference, then formula (2) corresponding expression can be written as

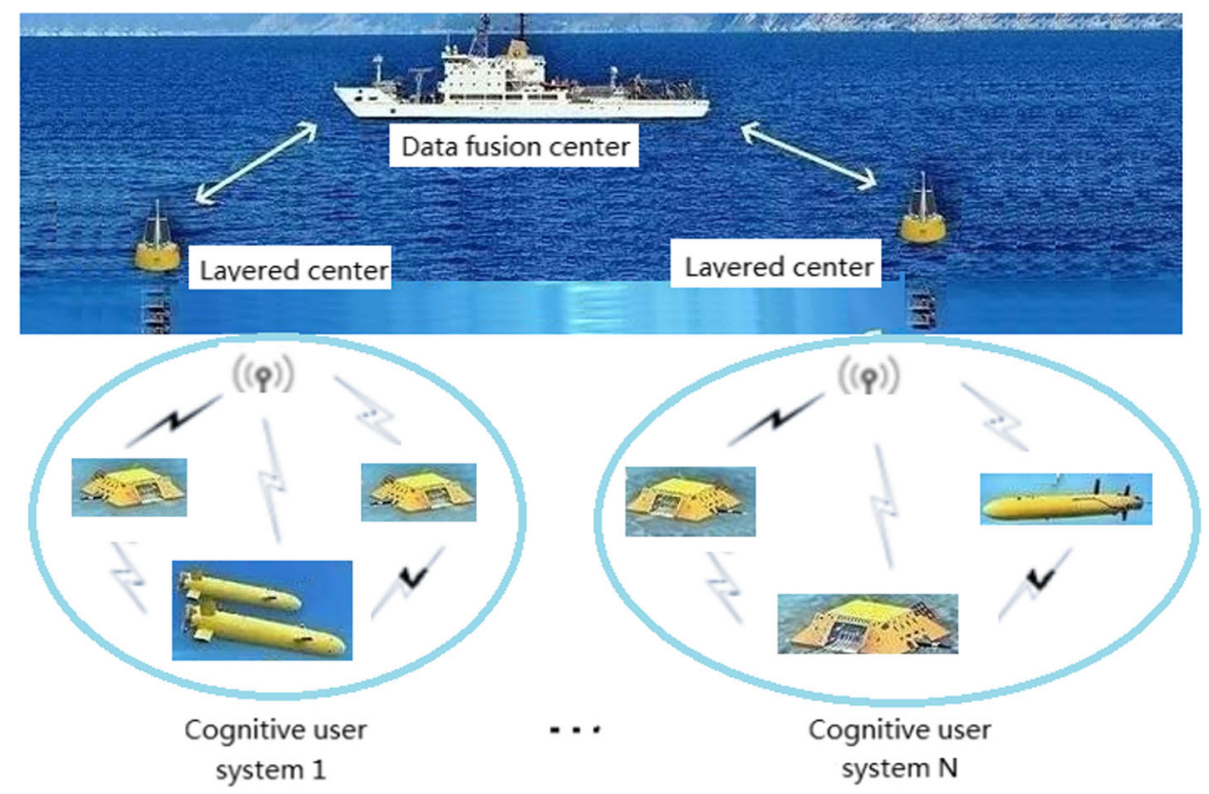

Fig. 1 Multi-layer structure model of an underwater communication system block diagram. When the signal detection is required, the control center will issue an indication signal, and all members of the grading center will perform the signal detection operation synchronously. Each SU collects the compressed data, uses the Bayesian model to obtain the shared super-parameters, and transmits the results to the cognitive radio user base station to obtain the preliminary signal detection results. When members of the hierarchical center collect perceptual data, distributed computing is performed and the results are broadcast to the hierarchical control center. After a lot of information exchange, the frequency spectrum of the spectrum signal detection algorithm is proposed to be close to a fixed value, the next step is through the data fusion center on the individual level data fusion, the center of the implement multiple layers joint spectrum signal detection, this paper puts forward a kind of based on Dirichlet process (DP) hierarchical Bayesian model to achieve compression perception. The data are grouped and the shared superparameters are mined in each group to obtain the optimal signal detection results 


$$
y=\Phi X+\Phi W
$$

where $w=\Phi W$, and assuming underwater noise that the average value of the service is 0 , the Gaussian distribution of variance $\sigma^{2}$, and make the parameter $\lambda_{0}=\frac{1}{\sigma^{2}}$, then the observed value $y$ from the Gaussian distribution. The likelihood function of the observed value $y$ can be expressed as:

$$
p\left\{y \mid X, \lambda_{0}\right\}=\left(2 \pi / \lambda_{0}\right)^{-\frac{M}{2}} \exp \left(-\frac{\lambda_{0}}{2}\|y-\Phi X\|_{2}^{2}\right)
$$

Suppose that the signal sent by the cognitive radio user $X$ is distributed from a Gaussian with a mean of zero, one-dimensional discrete-time signal $X=\left[x_{1}, X_{2} \ldots\right.$ $\left.x_{N}\right]$, the $k$ discrete value is denoted by $X_{k}$. The likelihood function of the discrete-time signal is as follows:

$$
p\left\{X \mid \lambda, \lambda_{0}\right\}=\prod_{k=1}^{M} N\left(X_{k} \mid 0, \lambda_{0}^{-1}, \lambda_{k}^{-1}\right)
$$

Among them, $M$ is the number of sampling points in the cognitive radio user's compressed perceptual data.

Among them, $\lambda=\left\{\lambda_{1}, \lambda_{2}, \cdots, \lambda_{M}\right\}$ is the super-parameter. According to the sparse characteristic of the wireless spectrum of underwater communication system, if the parameter $\lambda_{0}$ is assumed to be Gamma distribution, $X$ also has sparse property. Take $a$ and $b$ as parameters, namely:

$$
p\left\{\lambda_{0} \mid a, b\right\}=G a\left(\lambda_{0} \mid a, b\right)=\frac{b^{a}}{\Gamma(a)} \lambda_{0}^{a-1} \exp \left(-b \lambda_{0}\right)
$$

\section{Algorithm design}

For a hierarchical underwater communication cognitive radio network, assuming that there are $L$ cognitive radio users in the hierarchical center, the compressed sensing model provided extends to all cognitive radio users in the layer.

$$
\left\{\begin{array}{c}
y_{1}=\Phi_{1} X_{1}+w_{1} \\
\vdots \\
y_{L}=\Phi_{L} X_{L}+w_{L}
\end{array}\right.
$$

We assume that the variables of the Gaussian distribution are $X_{i}, y_{i}, w_{i}$, and $\Phi_{i}$ is the $M \times N$ dimension variable, where $i=1,2, \ldots L$. The likelihood function of the single cognitive radio user observation value in the hierarchical center can be expressed as:

$$
p\left\{y_{i} \mid X_{i}, \lambda_{0}\right\}=\left(2 \pi / \lambda_{0}\right)^{-\frac{m_{i}}{2}} \exp \left(-\frac{\lambda_{0}}{2}\left\|y_{i}-\Phi_{i} X_{i}\right\|_{2}^{2}\right)
$$

$m_{i}$ - - the number of points in the compressed sensing data of the $i$ th cognitive user.

In the underwater communication cognitive wireless network, the data of single hierarchical center perception is combined signal detection through the fusion center
[15]. Based on compress sensing, automatic grouping of compressed perceptual data for hierarchical Bayesian model is realized, different stratified centers may have different hyper-parameter: $\lambda=\left\{\lambda_{i 1}, \lambda_{i 2}, \cdots, \lambda_{i M}\right\}$, and $i=1$, $2, \cdots, C(C$ is the number of stratified centers in CRN). It is assumed that $\left\{\lambda_{i}, i=1,2, \cdots, C\right\} G$ is independent of the same distribution and $G$ is one of DP implementations. When the fusion center obtains the super-parametric information $\lambda^{-\mathrm{i}}=\left\{\lambda_{1}, \lambda_{2}, \cdots, \lambda_{i-1}, \lambda_{i+1}, \ldots \lambda_{c}\right\}$ of other stratified centers, the underlying distribution G0 will be updated. which is as follows:

$$
\begin{aligned}
p\left(\lambda_{i} \mid \lambda^{-i}, \xi, G_{0}\right)= & \frac{\xi}{\xi+C-1} G_{0} \\
& +\frac{1}{\xi+C-1} \sum_{k=1}^{K} n_{k}^{-i} \delta_{\lambda_{K}}
\end{aligned}
$$

where $\delta_{\lambda_{K}}$ is a pulse function. If $n_{k}^{-i}$ is used to denote the number of subsets with different values in the superparametric set $\left\{\lambda_{k}\right\}_{k=1}^{C}$ of hierarchical centers. It is shown that the new hyper-parameter $\lambda_{\mathrm{k}}$ is more inclined to be selected the larger membership $n_{k}^{-i}$ when implemented by DP.

In the data fusion center, if the super-parameter is represented by " $\lambda$ " and the $K$ super-parameter is represented by $\lambda_{k}^{*}$, the probability of the distribution $G$ is expressed $l_{k}$ at the point $\lambda_{k}^{*}$. Moreover, $\sum_{k=1}^{J} l_{k}=1, J$ is the number of possible values of super-parameters [16], $J<<C$. In the DP-based hierarchical Bayesian model, the maximum likelihood function corresponding to the data fusion center $\lambda^{*}$ can be further expressed as $l\left(\lambda^{*}\right)$ $=\sum_{k=1}^{J} \ell_{k}\left(\lambda_{k}^{*}\right)$, if the probability distribution is represented as the $j$ th stratified center and the $k$ th $\lambda_{k}^{*}$ is taken as the maximum super-parameter, then:

$$
\begin{aligned}
\ell_{k}\left(\lambda_{k}^{*}\right) & \left.=\sum_{j=1}^{C} \gamma_{j, k} \log p\left(y_{j} \mid \lambda_{k}^{*}\right)\right] \\
& \left.=\sum_{j=1}^{C} \gamma_{j, k} \log \int p\left(y_{j} \mid X_{j}, \lambda_{0}\right) p\left(y_{j} \mid \lambda_{k}^{*}, \lambda_{0}\right) p\left(\lambda_{0} \mid a, b\right) d X_{j} d \lambda_{0}\right] \\
& =-\frac{1}{2} \sum_{j=1}^{C} \gamma_{j, k}\left[\left(m_{j}+2 a\right) \log \left(y_{j}^{T} \Lambda_{j, k}^{-1} y_{j}+b\right)+\log \left|\Lambda_{j, k}\right|\right]+\text { Const }
\end{aligned}
$$

Where

$$
\begin{aligned}
\Lambda_{j, k} & =\mathbf{E}+\sum_{t=1, t \neq n}^{M} \lambda_{k, t}^{*-1} \Phi_{j, t} \Phi_{j, t}^{T}+\alpha_{k, n}^{*-1} \Phi_{j, n} \Phi_{j, n}^{T} \\
& =\Lambda_{j, k,-n}+\lambda_{k, n}^{*-1} \Phi_{j, n} \Phi_{j, n}^{T}
\end{aligned}
$$

where $\Lambda_{j, k}$ is not included in the $\Lambda_{j, k}$ where is the part of the $n$ column vector $\left(\Phi_{j, k, n}\right)$ that does not contain $\Phi_{j, k}: n=1,2 \cdots, M$. The determinant and inverse matrix of $\Lambda_{j, k}$ can be further expressed as: 


$$
\begin{aligned}
& \left|\Lambda_{j, k}\right|=\left|\Lambda_{j, k,-n} \| 1+\lambda_{k, n}^{*-1} \Phi_{j, n}^{T} \Lambda_{j, k,-n}^{-1} \Phi_{j, n}\right| \\
& \Lambda_{j, k}^{-1}=\Lambda_{j, k,-n}^{-1} \frac{\Lambda_{j, k,-n}^{-1} \Phi_{j, n} \Phi_{j, n}^{T} \Lambda_{j, k,-n}^{-1}}{\lambda_{k, n}^{*} \Lambda_{j, k,-n}^{-1} \Phi_{j, n}}
\end{aligned}
$$

In this case, $\lambda_{N, k}^{*}$ represents the Kth stratified center, the $n$th super-parameter. When the partial derivative with respect to $\frac{\partial l_{k}\left(\lambda_{k}^{*}\right)}{\partial \lambda_{j, k}}=0$, the extreme point can be obtained, namely:

$$
\lambda_{j, k} \approx \frac{L}{\sum_{i=1}^{L} \frac{\left(m_{i}+2 a\right) h_{j, k}^{2} / t_{j, k}-p_{j, k}}{p_{j, k}\left(p_{j, k}-h_{j, k}^{2} / t_{j, k}\right)}}
$$

Assuming that:

$$
\begin{aligned}
& p_{j, k}=\Phi_{\mathrm{j}, k}^{T} \Lambda_{\mathrm{j}, \mathrm{k}}^{-1} \Phi_{\mathrm{j}, \mathrm{k}} \\
& h_{j, k}=\Phi_{\mathrm{i}, k}^{T} \Lambda_{\mathrm{i}, \mathrm{k}}^{-1} y_{i} \\
& t_{j, k}=y_{i}^{T} \Lambda_{\mathrm{j}, \mathrm{k}}^{-1} y_{i}+2 \mathrm{~b}
\end{aligned}
$$

If the denominator is 0 , then $\lambda_{j, k}=\infty$. It needs to be removed $\Phi_{j, k}$. So we can use the formula (12) and $\lambda_{j}$, ${ }_{k}=\infty$. Update the super-parameters $\lambda_{j, k}$ continuously, $\{k=1,2 \ldots L, j=1,2 \ldots N\}$.

In order to obtain the optimal super-parameter, the detailed steps of the proposed algorithm are as follows:

(1) Initialization parameter $\lambda_{j, k}$ and base vector $\Phi_{i}$, $\{k=1,2 \ldots L ; i=1,2, \ldots, N ; j=1,2 \ldots N\}$;

(2) For any $k=1,2 \ldots L$, you pick a basis vector $\Phi_{j, k}$ and calculate the mean $\mu_{i}$ and the covariance $\Sigma_{i}$ and calculate the following:

$$
\begin{aligned}
& P_{j, k}=\Phi_{\mathrm{j}, k}^{T} \Lambda_{\mathrm{j}}^{-1} \Phi_{\mathrm{j}, \mathrm{k}} \\
& H_{j, k}=\Phi_{\mathrm{i}, k}^{T} \Lambda_{\mathrm{i}}^{-1} y_{i} \\
& T_{j, k}=y_{j}^{T} \Lambda_{\mathrm{j}}^{-1} y_{j}+2 b
\end{aligned}
$$

If $p_{j, k}=P_{j, k}+\frac{P^{2} j, k}{\lambda_{k}-P_{j, k}}, \quad h_{j, k}=H_{j, k}+\frac{P_{j, k} H_{j, k}}{\lambda_{n}-P_{j, k}}$, and $t_{j, k}$ $=T_{j, k}+\frac{H_{j, k}^{2}}{\lambda_{n}-P_{j, k}}$. According to formula (15) to update the super-parameter $\lambda_{j, k}$, when selecting the super-parameter, the increment $\Delta l_{k}\left(\lambda_{j, k}\right)$ of likelihood function $l_{k}\left(\lambda_{j, k}\right)$ should be maximized during each iteration;

(3) When the number of iterations is greater than the set threshold or when $\Delta l_{k}\left(\lambda_{j, k}\right)$ is less than a certain value, reach the termination condition of iteration algorithm and stop iteration; otherwise, go back to step
(2). The final decision can be made by comparing the obtained optimal over-parameter with the preset threshold value.

The final decision is realized by the iterative algorithm, which initializes parameters $\lambda_{k, n}^{*}$ and base vectors $\Phi_{j, n}$ and constantly updates weight coefficients $\gamma_{j, k}$ and $\lambda_{k, n}^{*}$. The super-parameters $\lambda_{k, n}^{*}$ were selected in each iteration process to maximize the increment until the calculated increment reaches the iteration termination condition or the iteration number reaches the prescribed upper limit; otherwise, the parameters are updated continuously until the iteration algorithm stops. The binary spectrum decision result is obtained by using the obtained superparameters and the preset threshold value.

\section{Simulation analysis}

The simulation conditions are as follows: in the underwater communication system cognitive network, the single-layer cognitive radio network numbers are 50 , and each layer has 20 SUs. The whole wireless spectrum is evenly divided into 200 subcarriers, and there are $50 \mathrm{PU}$ in the network. Each PU communication takes up one subcarrier frequency and sets the noise of underwater communication system which is Gaussian white noise. Performance analysis and simulation are as follows.

\subsection{Complexity analysis}

The new cooperative spectrum signal detection algorithm for underwater communication based on compressed sensing is to introduce the unknown variable to a certain prior condition distribution, select and update the hyper-parameter through the basis function, and increase continuously through the iterative operation until the iteration condition is met. The observation matrix only contains the basis function existing in the current model. The various algorithm complexity pairs are shown in Table 1. In the process of solving the maximum likelihood function, comparing the EM algorithm, the complexity of $O\left(N^{3}\right)$, the matching pursuit (MP) [17, 18], and orthogonal matching pursuit (OMP) algorithms [19], the complexity of $O(M \times N)$ adopts the algorithm mentioned and only updates the super-parameter at a time, and to achieve some estimate parameter and error covariance update, eliminating the complex matrix inversion process,

Table 1 Comparison of algorithm complexity

\begin{tabular}{ll}
\hline Algorithm & Complexity \\
\hline EM & $O\left(N^{3}\right)$ \\
MP & $O(M \times N), M \geq N$ \\
OMP & $O(M \times N), M \geq N$ \\
Paper algorithm & Less than $O(N \times N)$ \\
\hline
\end{tabular}


the dimension of point estimate parameters, and covariance dimension is far less than $O(N \times N)$ in sparse signal, so the computational complexity of compressed sampling is effectively reduced.

\subsection{Noise robustness}

In broadband spectrum signal detection of the underwater communication system, there are all kinds of interference in the channel, and the performance of the algorithm will also be affected by the underwater noise. But from the broadband spectrum collaborative perception algorithm based on compression perception, we found in the derivation that updating the parameters of the iterative process has nothing to do with the noise variance, $\lambda_{0}=\frac{1}{\sigma^{2}}$, so this section of perception algorithm can effectively reduce the influence of underwater noise to eliminate noise variance SNR wall of the problems of uncertainty and to improve the accuracy of the detection algorithm. The performance of the algorithm is verified at different underwater noise levels. In Gaussian white noise, Fig. 2 depicts the normalization error performance of different algorithms. The comparison shows that the novel cooperative spectrum signal detection algorithm based on compressed sensing for underwater communication system has advantages over other algorithms under the given noise level.

\subsection{Sensing performance analysis}

\subsubsection{Relationship between SNR and detection probability}

In the underwater communication system, assume that each primary user sends a signal length of $N=256$ and the number of samples is $M=30$. Cognitive users can exchange information with adjacent users. Considering the signal-to-noise ratio varies from -15 to $5 \mathrm{~dB}$, we can compare the detection probabilities of paper algorithm and the classical algorithms when the number of underwater communication cooperative users is different. After the cognitive user obtains the local detection result through the compressed sensing, the detection probability can be obtained according to the actual spectrum occupancy of the channel and the simulated spectrum occupancy. The simulation result is shown in Fig. 3.

It is clear that our method can present a higher detection probability than that of the traditional method with the aid of the increasing number of underwater communication cooperative users. However, whether it is a single-user or multiple user detection, it is not difficult to find that when the signal-to-noise ratio is very low, between $-15 \mathrm{~dB}$ and $10 \mathrm{~dB}$, the detection probability is very low and the performance of cooperative spectrum detection do not have many advantages. This low signalto-noise ratio state is equivalent to a situation where severe shadow-fading or other fading are encountered in

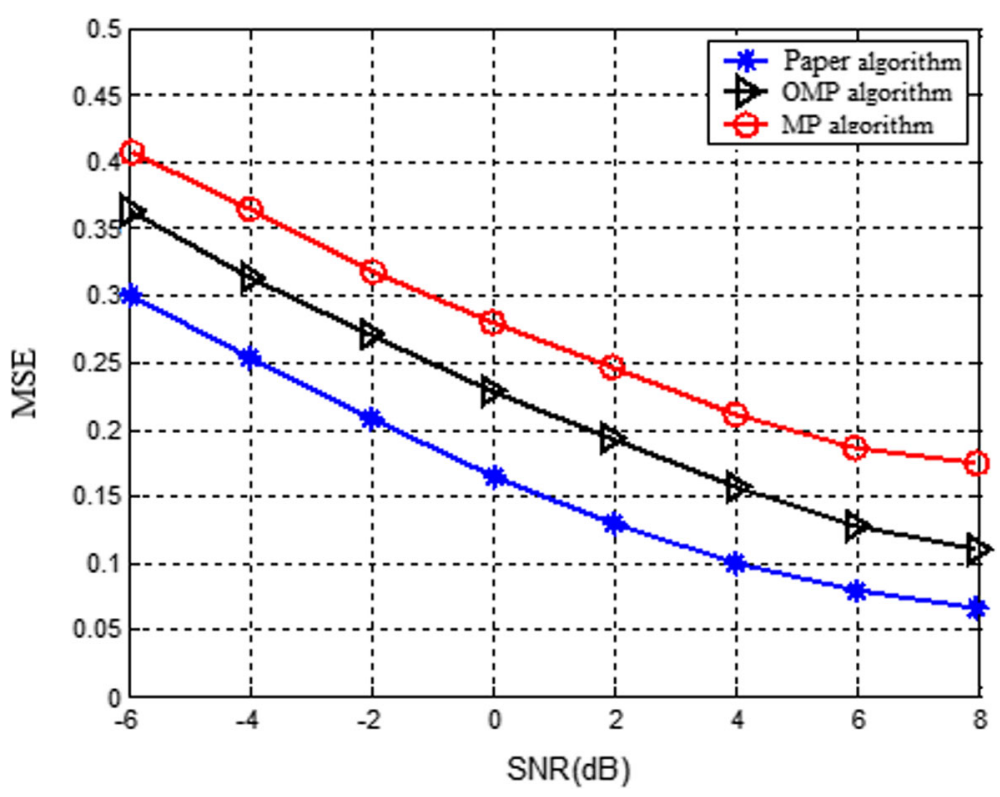

Fig. 2 Normalized error performance of different algorithms under white Gaussian noise. In the wideband spectrum signal detection, there are various kinds of interference in the channel, and the performance of the algorithm is also affected by the noise. However, from the derivation of the wideband spectrum cooperative perception algorithm based on compressed sensing, we find that the parameters of the updating iterative process have nothing to do with noise variance, so this part of the perception algorithm can effectively reduce the impact of noise, eliminate the uncertainty of noise variance SNR, and improve the accuracy of the detection algorithm. At different noise levels, the performance of the algorithm is verified. In Gaussian white noise, Fig. 2 describes the normalized error performance of different algorithms. The comparison shows that the paper algorithm based on compressed sensing has advantages over other algorithms at a given noise level 


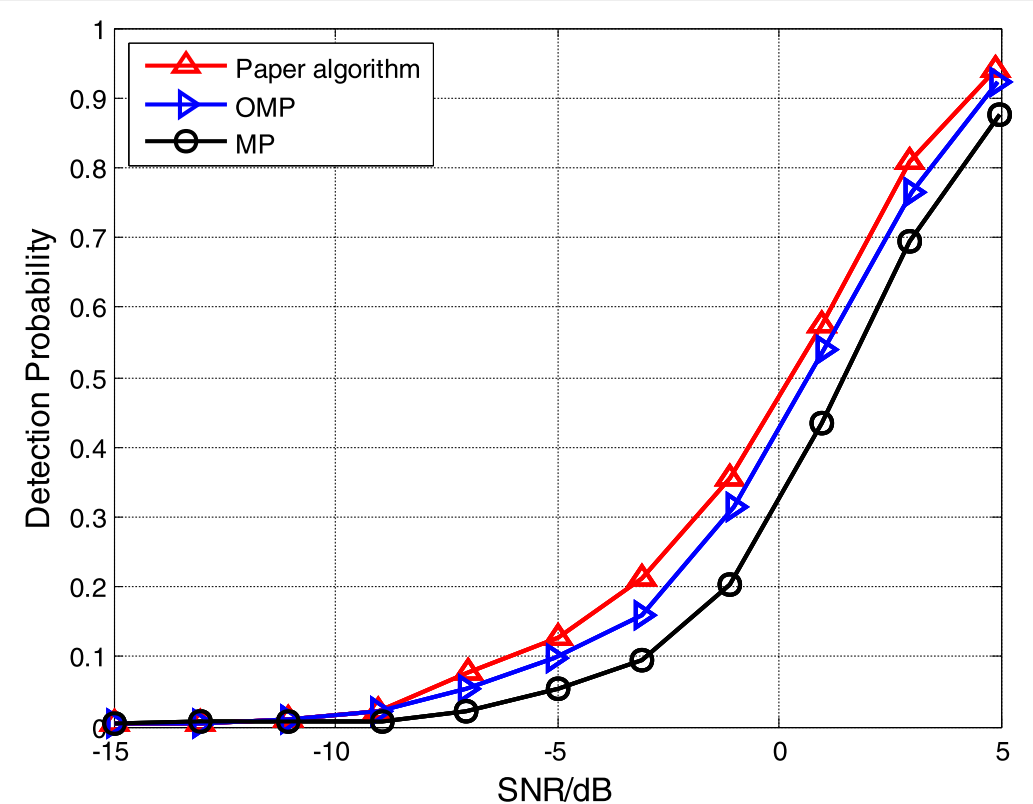

Fig. 3 The curve of the relationship between SNR and detection probability. Considering the change of SNR between - $15 \mathrm{db}$ and $5 \mathrm{~dB}$, we can compare the detection probability of the paper algorithm and the classical algorithm in the case of different number of cooperative users. After the cognitive user obtains the local detection results through compressed sensing, the detection probability is obtained according to the actual spectrum occupation of the channel and the simulated spectrum occupation. With the increase of the number of cooperative users, our method has higher detection probability than the traditional method. However, no matter it is single-user detection or multi-user detection, it is not difficult to find that when the SNR is very low, the detection probability is very low between $-15 \mathrm{db}$ and $10 \mathrm{~dB}$, and the performance of cooperative spectrum detection does not have many advantages. This low SNR state is equivalent to a severe shadow-fading or other fading in real environments. Cognitive users in this environment will affect the performance of cooperative spectrum detection and even reduce the detection performance of cognitive users with high SNR. Therefore, synergetic spectrum detection not only improves the detection performance, but also reduces it

the actual underwater communication environment. Cognitive users in this environment will affect the performance of collaborative spectrum detection and even reduce the detection performance of cognitive users in underwater acoustic channel state with high SNR. So, the cooperative spectrum detection is helpless to improve the performance, but reduces detection performance.

\subsubsection{The relation between sampling number and detection probability}

If each $\mathrm{SU}$ of the underwater communication system collects different observations through compressed sensing, we can further simulate its operating characteristic curve.

This result in Fig. 4 indicates that the spectrum sensing performance of the MP algorithm and the OMP algorithm is gradually improved when the number of observation points of the compressed sensing is increased. This is because that the more sampled values, the more information is obtained. Besides, the MP algorithm and the OMP algorithm are algorithms for a single task, regardless of the spatial diversity information, and the only information available is from the compressed sensing observations. Compared with the MP and OMP algorithms, the proposed algorithm has no change in the ROC curve when the number of observation is greater than 20 and the detection probability approaches 1 , because it makes full use of the compressed sensing data information collected by each SU and the number of iterations of the algorithm is not related to the number of samples. Even at lower sample values, it can still obtain better detection performance and effectively reduce the system overhead of spectrum sensing. From the ROC of the three algorithms and considering the lower number of observation, it is obvious that in underwater communication system, the algorithm can achieve the purpose of effective signal detection through a small amount of information acquisition. Compared with traditional MP and OMP, the performance of underwater acoustic communication system is improved.

\subsubsection{The relation between false alarm probability and detection probability}

To better verify the effectiveness of the novel cooperative spectrum signal detection algorithm for the underwater communication system, we compare it to existing legacy and improved algorithms. In paper [20], a sparse Bayesian learning algorithm is proposed to achieve local 


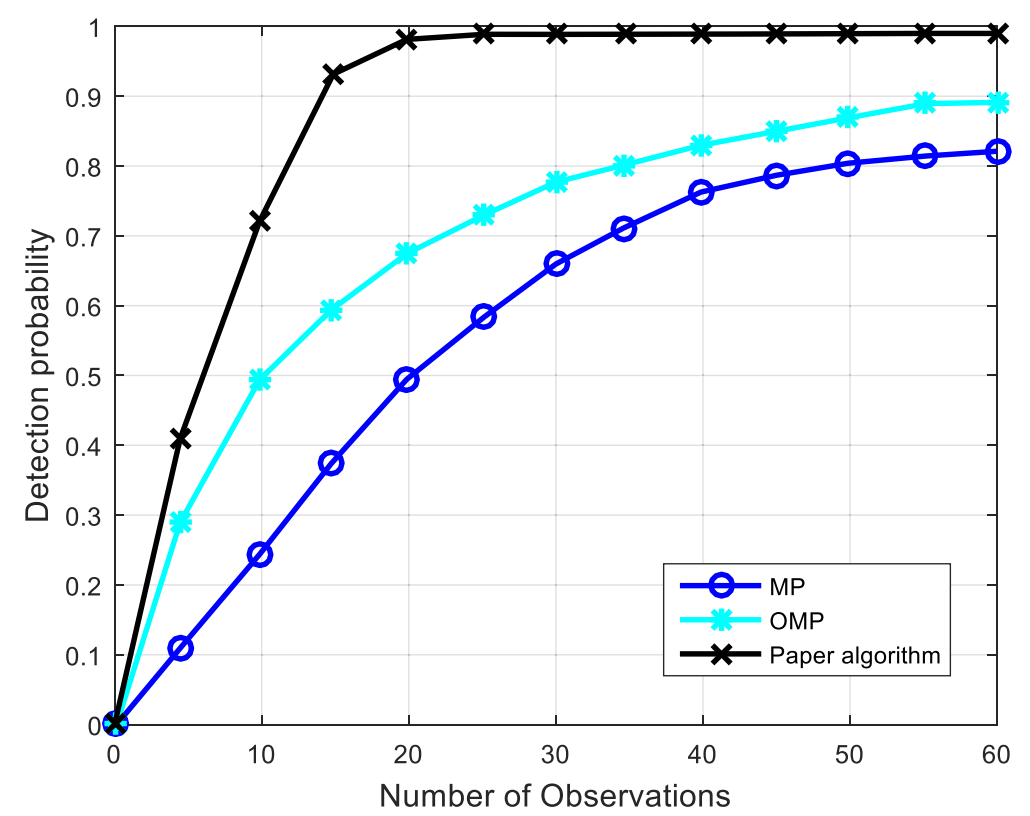

Fig. 4 The curve of the relationship between observed values and detection probability. This result in Fig. 4 indicates that the spectrum sensing performance of the MP algorithm and the OMP algorithm is gradually improved when the number of observation points of the compressed sensing is increased. This is because that the more sampled values, the more information is obtained. Besides, the MP algorithm and the OMP algorithm are algorithms for a single task, regardless of the spatial diversity information, and the only information available is from the compressed sensing observations. Compared with the MP and OMP algorithms, the proposed algorithm has no change in the ROC curve when the number of observation is greater than 20 and the detection probability approaches 1. Because it makes full use of the compressed sensing data information collected by each SU and the number of iterations of the algorithm is not related to the number of samples. Even at lower sample values, it can still obtain batter detection performance and effectively reduce the system overhead of spectrum sensing. From the ROC of the three algorithms and considering the lower number of observation, it is obvious that the correct detection probability of the paper algorithm is better than the MP and OMP algorithms, and the spectrum sensing performance is better

optimization through sparse linear regression using the mechanism of support vector machine, so as to complete the single-task Bayesian compressed sensing algorithm (SBCS) for solving the process. In paper [21], a Wide-Band Cooperative Compressive signal detection for Cognitive Radio Systems Using Distributed Sensing Matrix (WBCCSS) is proposed. This algorithm introduces the distributed perception matrix method and uses the distributed multi-node fusion to complete the spectrum perception. In paper [22], this has been put forward by building a hierarchical Bayesian model of multitasking, and assuming that the cognitive user acquisition of compressed sensing data with the same parameters, by the fusion center data compression perception of the cognitive users to seek the optimal share of superparameter and thus to accomplish the signal reconstruction process of multitasking compression sensing algorithm (MTCS).

Figure 5 indicates the four algorithms under the same SNR performance simulation curve comparison as follows: when the false alarm probability is 0.1 , the detection probabilities of BCS, WBCCSS, MTCS, and paper algorithm were $0.3328,0.3815,0.5246$, and 0.7925 , respectively, so the joint spectrum signal detection algorithm compared to BCS, WBCCSS, MTCS algorithm, and spectrum detection has better performance, and from the simulation curve, it can be found using the Dirichlet process that it can be achieved within each group shared with shearing section at parameters. The process fully considers the perception of the temporal correlation of the data compression. The performance of the algorithm is obviously better than BCS, WBCCSS, and MTCS.

\section{Results and discussion}

This paper presents an efficient underwater communication signal detection method based on compressed sensing technology. In underwater communication cognitive wireless networks, different cognitive radio users and authorized users may have different sparse spectrum due to their different distributed environments in different spaces and the complexity of underwater channels. In the algorithm design, each CR node uses compressed sampling technology to estimate the frequency spectrum to reduce the rate and overhead of sonar signal sampling. The Bayesian model is used to find the optimal super-parameter to detect the spectrum information. The proposed algorithm effectively solves the high computational complexity of sonar signal reconstruction in compression sensing. The joint cooperative spectrum 


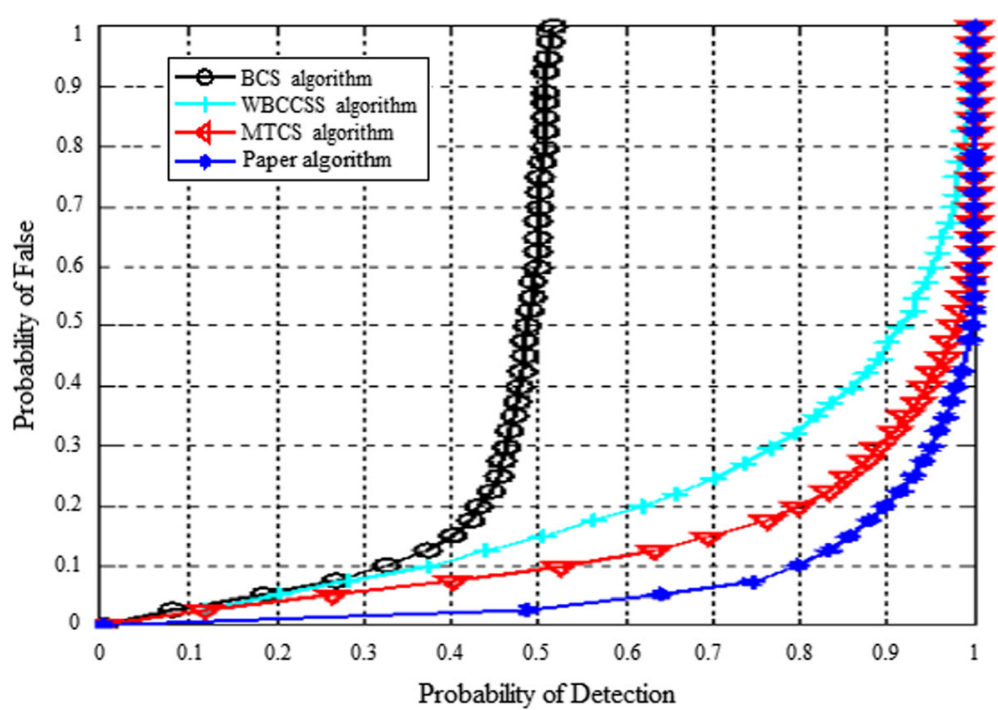

Fig. 5 Four algorithms' performance comparison under the same SNR. Figure 5 indicates the four algorithms under the same SNR performance simulation curve comparison as follows: when the false alarm probability is 0.1 , the detection probabilities of BCS, WBCCSS, MTCS, and paper algorithm were $0.3328,0.3815,0.5246$, and 0.7925 , so the joint spectrum signal detection algorithm compared to BCS, WBCCSS, MTCS algorithm, and spectrum detection has better performance, and from the simulation curve, it can be found using the Dirichlet process that this can be achieved within each group shared with shearing section at parameters. The process fully considers the perception of the temporal correlation of data compression. The performance of the algorithm is obviously better than BCS, WBCCSS, and MTCS

algorithm utilizes the information of non-parametric grouping mechanism. The multi-layer Bayesian model introduces the Dirichlet process to realize the automatic grouping of compressed perceptual data, deduces the shared hyper-parameter, then selects the best superparameter to decide the spectrum through the fusion center, and finally transfers it to the SU. The algorithm makes full use of compressed perceptual data collected by different cognitive radio users in different layers and performs fusion and collaboration to complete signal detection and effectively improve the performance of underwater communication spectrum signal detection. Effectively reduce the channel overhead in the underwater communication system; solve the series of problems such as high complexity, slow convergence speed, and low reconstruction precision of the compressed sensing reconstruction algorithm; improve the efficiency; and increase the throughput of the system, which has a great significance for achieving high-quality underwater communication.

From the current research status, there are still the following problems and challenges in the wide-band spectrum compressed sensing technology of underwater communication system: at present, spectrum detection is carried out by using compressed sensing, and the processing method in most literatures is to obtain the compressed sampling first, then reconstruct the sonar signal, and finally carry out spectrum detection on the reconstructed signal. Its perceptual performance is easily affected by the variance uncertainty of noise. The compressed sensing reconstruction algorithm is a np-hard problem with high computational complexity and needs more time, especially for the broadband signal reconstruction process which takes too long and is not conducive to real-time application. Therefore, how to effectively reduce the reconstruction time and the reconstructed signal with a good mean square error is a big difficult problem in compressed sensing.

Secondly, as the sonar signal sparsity in the actual underwater communication network is unknown and variable, it is difficult to determine the signal sparsity for some complex sonar signals, or it is not precisely sparse. In order to ensure the reconstruction accuracy of broadband sonar signals, the sampling rate usually meets the condition of the maximum possible sparsity of the signals, resulting in the waste of sampling resources. Moreover, in CR networks, the cooperative compressed spectrum sensing fusion mechanism is crucial, which will directly affect the performance of underwater cognitive wireless networks. The corresponding efficient and low-complexity fusion mechanism still needs to be further explored.

\section{Abbreviations}

BCS: Bayesian compressed sensing; CR: Cognitive radio; CRN: Cognitive radio network; DP: Dirichlet process; EM: Expectation maximization; MP: Matching pursuit; MTCS: Multitasking compression sensing; OMP: Orthogonal matching pursuit; PU: Perception of the user; RIP: Restricted isometry property; RN: NDimensional Real Vector Space; ROC: Receiver operating characteristic curve; SBCS: Single-task Bayesian compressed sensing; SNR: Signal-to-noise ratio; SU: Secondary user; WBCCSS: Wide-Band Cooperative Compressive Spectrum Signal 


\section{Acknowledgements}

The authors acknowledged the anonymous reviewers and editors for their efforts in valuable comments and suggestions.

\section{Authors' contributions}

$J X$ proposes the innovation ideas and theoretical analysis. The other authors also have contributed jointly to the manuscript. All authors have read and approved the final manuscript.

\section{Funding}

This work was supported in part by the Heilongjiang Province Natural Science Fund Project (No. F2015019) and Heilongjiang Provincial Postdoctoral Science Foundation (No. LBH-Z16054).

\section{Availability of data and materials}

Data sharing is not applicable to this article as no datasets were generated or analyzed during the current study.

\section{Competing interests}

The authors declare that they have no competing interests.

\section{Author details}

${ }^{1}$ Harbin Institute of Technology, Harbin 150000, Heilongjiang, China

${ }^{2}$ Heilongjiang University of Science and Technology, 150000, Harbin, China.

${ }^{3}$ Heilongjiang Province Public Security Department, 150000, Harbin, China.

Received: 4 April 2019 Accepted: 10 September 2019

Published online: 15 October 2019

\section{References}

1. J. Verlant, A. Bourdoux, J. Dricot, P. De Doncker, in International Conference on Computing. Networking and Communications. Wideband compressed sensing for cognitive radios using optimum detector with no reconstruction (2012), pp. 887-891. https://doi.org/10.1109/ICCNC.2012.6167552

2. C. Li, F. Zeng, Z. Tian, Distributed compressive spectrum sensing in cooperative multihop cognitive networks. IEEE J. Sel. Top. Sign. Proces. 5(1), 37-48 (2011). https://doi.org/10.1109/JSTSP.2010.2055037

3. M.A.T. Figueiredo, R.D. Nowak, S.J. Wright, Gradient projection for sparse reconstruction: Application to compressed sensing and other inverse problems. IEEE J. Sel. Top. Sign. Proces. 1(4), 586-598 (2007). https://doi.org/ 10.1109/JSTSP.2007.910281

4. L. Jia, W. Qiang, S. Yi, in Instrumentation and Measurement Technology Conference (I2MTC). Performance analysis of OMP algorithm in recovering magnitude decaying sparse signals in compressive sensing (2013), pp. 1781-1785. https://doi.org/10.1109/I2MTC.2013.6555721

5. D. David, T. Yaakov, D. Iddo, Sparse solution of underdetermined linear equations by stagewise orthogonal matching pursuit. Tech. Rep. 3(9), 1-39 (2006). https://doi.org/10.1109/tit.2011.2173241

6. L. Hongchao, J. Bo, L. Hongwei, B. Zheng, Superresolution ISAR imaging based on sparse Bayesian learning. Geosci. Remote Sens. 52(8), 5005-5013 (2013). https://doi.org/10.1109/TGRS.2013.2286402

7. G.P. Yang, X. Zhou, X.C. Yu, Sparse Bayesian model and correlation vector machine learning. Comput. Sci. 7(37), 225-228 (2010)

8. L. Poli, G. Oliveri, Bayesian compressive sensing approaches for the reconstruction of two-dimensional sparse scatterers under TE illuminations. Geosci. Remote Sens. 51(5), 2920-2936 (2012). https://doi. org/10.1109/TGRS.2012.2218613

9. S. Hong, Direct spectrum sensing form compressed measurements. IEEE MILCOM 52(10), 1187-1192 (2010). https://doi.org/10.1109/ MILCOM.2010.5680103

10. Y. Huogen, T. Wanbin, Optimization of multiple-channel cooperativesignal detection with data fusion rule in cognitive radio networks. J. Electron. 29(6), 515-522 (2012). https://doi.org/10.1007/s11767-012-0863-2

11. H. Hu, H. Zhang, H. Yu, Throughput-delay trade-off for cognitive radio networks: A convex optimization perspective. Abstr. Appl. Anal. 14(1), 1-8 (2014)

12. E.C.Y. Peh, Y.C. Liang, Y.L. Guan, Optimization of cooperative sensing in cognitive radio networks: A sensing-throughput tradeoff view. IEEE Trans. Veh. Technol. 58(9), 5294-5299 (2009). https://doi.org/10.1109/WCNC.2007.11

13. J. Xiaolin, A joint cooperative spectrum signal detection algorithm based on compressed sensing. J. Shanghai Jiaotong Univ. 47(7), 1099-1103 (2013)
14. J.N. Laska, P.T. Boufounos, Democracy in action: Quantization, saturation and compressive sensing. Appl. Comput. Harmon. Anal. 31(3), 429-443 (2011). https://doi.org/10.1016/j.acha.2011.02.002

15. X. Liu, F. Li, Z. Na, Optimal resource allocation in simultaneous cooperative spectrum sensing and energy harvesting for multichannel cognitive radio. IEEE ACCESS 5, 3801-3812 (2017). https://doi.org/10. 1109/ACCESS.2017.2677976

16. W. FangQing, Z. Gong, B. De, A multi-task compressed sensing reconstruction algorithm based on block sparse Bayesian learning. Acta Phys. Sin. 64(7), 70201-70206 (2015). https://doi.org/10.7498/aps.64.070201

17. L. Rongpeng, Z. Zhifeng, Adaptive multi-task compressive sensing for localisation in wireless local area networks. IET Commun. 8(10), 1736-1744 (2014). https://doi.org/10.1049/iet-com.2013.1019

18. L. Xin, J. Min, Z. Na, Multi-modal cooperative spectrum sensing based on Dempster-Shafer fusion in 5G-based cognitive radio. IEEE ACCESS 6(99), 199-208 (2018). https://doi.org/10.1109/ACCESS.2017.2761910

19. L. Xin, Z. Xueyan, J. Min, 5G-based green broadband communication system design with simultaneous wireless information and power transfer. Phys. Commun. 28, 130-137 (2018). https://doi.org/10.1016/j.phycom.2018.03.015

20. S. Ji, Y. Xue, L. Carin, Bayesian compressive sensing. IEEE Trans. Signal Process. 56(6), 2346-2356 (2008). https://doi.org/10.1109/tsp.2007.914345

21. X.L. Huang, F. Hu, J. Wu, et al., Intelligent cooperative spectrum sensing via hierarchical Dirichlet process in cognitive radio networks. IEEE J. Sel. Areas Commun. 33(5), 771-787 (2015). https://doi.org/10.1109/JSAC.2014.2361075

22. L. Xue, H. Steven Siying, Bayesian compressed sensing based dynamic joint signal detection and primary user localization for dynamic spectrum access. Glob. Telecommun. Conf. Houst. Tex. USA, 611-619 (2011). https://doi.org/ 10.1109/GLOCOM.2011.6134530

\section{Publisher's Note}

Springer Nature remains neutral with regard to jurisdictional claims in published maps and institutional affiliations.

\section{Submit your manuscript to a SpringerOpen ${ }^{\circ}$ journal and benefit from:}

- Convenient online submission

Rigorous peer review

- Open access: articles freely available online

High visibility within the field

- Retaining the copyright to your article

Submit your next manuscript at $\boldsymbol{\nabla}$ springeropen.com 